\title{
Multimorbidität und geänderte Pharmakodynamik im Alter
}

Ein zentraler Aspekt der Pharmakotherapie im Alter ist die zunehmende Multimorbidität. Letztlich ist dies der Motor, der nicht nur über klassische Interaktionen, sondern auch über häufig nicht bedachte Bezüge sowohl die Wirksamkeit einer medikamentösen Therapie beeinflusst wie auch deren Risiken. Im Vortrag wird kurz auf typische multifaktoriell bedingte Risiken eingegangen, sowohl was epidemiologische Aspekte wie auch Aspekte der Pathophysiologie anbelangt. Obwohl sie als bedeutende klinische Fragestellung und Herausforderung allgemein Anerkennung findet, ist der theoretische Hintergrund der geriatrietypischen Multimorbidität nicht ausreichend beleuchtet und kommuniziert. Erstaunlicherweise gibt es nicht einmal eine anerkannte Konvention zur Definition der Multimorbidität. Im Vortrag wird daher ein Konzept zur geriatrietypischen Multimorbidität vorgestellt, welches sich stärker an der „pattern theory“ orientiert und Bezüge zur Pharmakotherapie daraus ableitet. Weiter verdient auch der Begriff Pharmakodynamik in diesem Zusammenhang eine Neubewertung und Öffnung für wesentliche, bisher aber nicht ausreichend berücksichtigte Aspekte, die an ausgewählten Beispielen beleuchtet werden. Damit ist in besonderer Weise die Ebene der funktionellen Ressourcen des Patienten angesprochen. Hierzu wird ein Schichtenmodell angeboten, in welchem unterschiedliche Effekte und Befunde verortet werden können und welches es erlaubt, analytische Prozesse in komplexen klinischen Situationen nachzuvollziehen und letztlich eine integrierende Betrachtung ermöglicht.
Interessenkonflikt

PD Dr. med. H. Burkhardt ist seit Jahren als Autor im FORTA-Konsortium engagiert sowie Mitglied der internationalen Interessengemeinschaft IGRIMUP zur Begrenzung der Polypharmazie. Sonst bestehen in diesem Zusammenhang keine Interessenkonflikte.

Autorinnen/Autoren

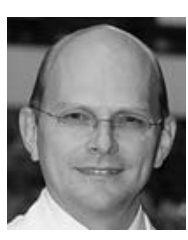

Heinrich Burkhardt

PD Dr. med., Direktor des Geriatrischen Zentrums, Universitätsklinikum Mannheim

Korrespondenzadresse

PD Dr. med. Heinrich Burkhardt Geriatrisches Zentrum Universitätsklinikum Mannheim Theodor-Kutzer-Ufer 1-3 68167 Mannheim heinrich.burkhardt@umm.de 